\title{
La política exterior española ante la inmigración rnagrebí*
}

\author{
Josep Ibáñez
}

Licenciado. Facultat de Ciències Políriques i de Sociologia

Investigadot asociado al Centre d'Estudis sobre la Pau i ef Desarmament

Universitat Autònoma de Barcelona. 08193 Bellaterra (Barcelona). Spain

\section{Resumen}

Los movimientos migratorios in:ernacionales son un fenómeno característico del Mediterráneo occidental que afecta a las políticas exteriores de los Estados receptores. Asf́, la política exterior española se ha visto especialmente influenciada por la inmigración magrebí, tanto en sus relaciones bilaterales con los países del Magreb como en ámbitos multilaterales, incluyendo en éstos su actuación en la Europa comunitaria y su contribución a diversos proyectos de cooperixción mediterránea.

Palabras clave: inmigración magrebi, polftica exterior espafiola, Mediterráneo occidental, seguridad internacional, Eutopa comunitaria.

\section{Abstract. The Spanish Foreign Policy facing Maghrebi Immigration}

International migration movements are a phenomenon characteristic of the Western Mediterranean affecting the foreign policies of the host states. Thus, Maghrebi immigration has had a special influence in the Spanish foreign policy, both in its bilateral relationships with the Maghreb countries and in multilateral spheres, including its diplomatic moves in the European Union and its contribution to various Mediterranean cooperation projects.

Key words: Maghrebi immigration, Spanish foreign policy, Western Mediterranean, international security, European Urion.

\section{Sumario}

El contexto internacional de Ensayos diplomáticos multilaterales la inmigración magrebí en Fspaña en el Meditertáneo

El estrechamiento de las relaciones economicas hispano-magrebtes

La polftica exterior y de seguridad E! filtzo de la Europa comunitaria Conclusiones española hacia el Mlagreb

Bibliograffa citada

* El presente artículo está parcialmente basado en la memoria de licenciatura uMigraciones y Relaciones Internacionales. La jolítica exterior española ante la inmigración magrebín, dirigida por Caterina Gardia y presentada en la Facultat de Ciencies Polítiques i de Sociologia de la UAB en septiembre de 1994 . 
Uno de los fenómenos internacionales que mayor atención ha captado entre expertos y responsables políticos de la cuenca mediterránea durante la última década ha sido el de las migraciones internacionales. Si el Mediterráneo es una región compleja, los movimientos migratorios que en ella tienen lugar también se caracterizan por una complejidad que no siempre se ha sabido tratar.

Los Estados implicados en este tratamiento, y particularmente los Estados receptores de inmigración, necesitan poner en funcionamiento políticas que afectan a varios ministerios gubernamentales y administraciones cuyas actuaciones requieren una coordinación difícil de alcanzar. Los ministerios tradicionalmente afectados por la inmigración eran los que se ocupaban de asuntos sociales, interior, trabajo, justicia, etc.; pero a partir de determinado momento, los recursos utilizados son insuficientes y cabe acudir a instrumentos de política exterior para complementar una acción con pretensiones de eficacia.

Desde esta perspectiva, las migraciones internacionales adquieren una dimensión y relevancia que las hace objeto de estudio por parte de la disciplina de las relaciones internacionales. Sin embargo, si bien es fácil encontrarse con investigaciones de tipo demográfico, sociológico, jurídico o histórico, escasos son los estudios con un carácter internacionalista. Globalmente, puede decirse que éstos han adoptado dos enfoques en el tratamiento de! fenómeno migratorio:

a) los migrantes en tanto que actores de las relaciones internacionales', por su capacidad de influir en la actuación política de un Estado, por su capacidad de "perforar" la soberanía estatal, según la formulación de Duchacek (1988: 5-6);

b) los efectos de la inmigración en la política exterior de los países receptores (Weiner, 1992: 91-126), en la mayoría de los casos concretados en la puesta en marcha de iniciativas políticas, económicas y diplomáticas, tanto a nivel bilateral como multilateral.

Precisamente desde esta segunda perspectiva plantearemos cuáles han sido las acciones llevadas a cabo por la política exterior española en relación con la inmigración procedente del Magreb. Pero antes de ello cabe enmarcar, siquiera brevemente, el fenómeno migratorio al que nos referimos.

\section{El contexto internacional de la inmigración magrebí en España}

Con todas las precauciones obligadas al generalizar, cuando utilizamos el término inmigración magrebí nos referimos a un colectivo humano que emigra de su lugar de origen, mayoritariamente, por motivos económicos. A tales migrantes económicos nos referiremos sin distinguir entre los que se encuentran en una situación legal o ilegal, aunque a muchos efectos la distinción no es banal. Es obligado señalar que la gran mayoría de los individuos en cues-

1. Sobre el concepto de actor internacional véase Carerina García Segura (1993: 13-31). 
tión proceden de Marruecos, quedando muy por dettás el número de argetinos, tunecinos, mauritanos y libios'.

La carencia de datos fiables y actualizados representa un problema estadistico importante a la hora de determinar la composición exacta de los inmigrados. Con todo, es posible apuntar algunas cifras orientativas. Así, en la Unión Europea de 1991 el porcentaje medio de población extranjera en situación legal procedente de países no comunitarios tondaba el 2,4\%, mientras que en España esa cifra apenas superaba el 1,2\% (Eurostat, 1993). Concretamente, las cifras de inmigrantes extranjeros legales no comunitarios en territorio español comienza.n a crecer a partir 1986, y las de procedencia magrebf ya habían comenzado a hacerlo en 1984, aunque el peso de estos últimos no deja de ser modesto, pues en 1989 tan sólo representaban el 3,9\% del total (Eurostat, 1991). Cabe destacar, sin embargo, que el nivel de inmigración irregular en España es elevado, alcanzando una tasa de irregularidad del $35 \%$ en 1988-1989, similar al de los otros nuevos países europeos de inmigracion ${ }^{3}$; y dentro del colectivo de inmigrantes irregulares, destacan por su número los nacionales de Marruecos, entre 60.000 y 100.000 individuos según las fuentes, como muestran los estudios del Colectivo Ioé (1987 / 1992). En cualquier caso, una estimación del total de extranjeros no comunitarios en España en 1989 representaría algo más del $2 \%$ sobre el total de población espanola, mientras que los magrebies no superarían en ningún caso el $0,3 \%$, una cifra muy modesta que no justifica alarmismos.

La contextualización de estos movimientos migratorios que tienen lugar desde mediados de los años ochenta hasta nuestros días pasa por las características geopolíticas del Mediterráneo occidental (Medoc). En este escenario se manifiestan claramente los contrastes Norte-Sur que justifican la utilización de términos como "frontera" o "fractura" para referirse al Medoc. Dichos contrastes son básicamente de tres tipos: a) demográficos, ya presentes en la actualidad, pero que por las dinámicas divergentes de las poblaciones ribereñas del norte y del sur mostrarán mayores diferencias en los próximos treinta años ${ }^{4}$; b) económicos, enraizados en estructuras productivas muy diferentes que están en el origen de la disparidad entre las condiciones de vida de las orillas norte y sur, y uno de cuyos efectos es la atracción de las poblaciones magrebíes por el bienestar del suroeste de Europa, y c) sociopolíticos, entendiendo en este caso divergencias en las formas de entender la sociedad y la política, algo muy influenciado por las concepciones religiosas sobre las que se asientan las civi-

2. Por Magreb entendemos el territorio que comprende Mauritania, Marruecos, Argelia, Túnez y Libia. Si bien la importancia de cada uno de estos Estados es muy variable desde el punto de vista de la emigración hacia Espafia, el Magreb suele enfocarse como una unidad geopolítica en la acción exterior espafiola.

3. La tasas de irregularidad de los otros páses meridionales de la Unión Etropea eran de 27,6\% en Grecia, 59,8\% en Italit y 38,8\% en Portugal (Isoplan, 1991).

4. Las variables más características de esta evolución divergente son la tasa de crecimiento demográfico, la estructura de la población, las tasas de actividad y el crecimiento de la población urbana. 
lizaciones. Precisamente, la ola de fundamentalismo religioso que se vive en algunos países islámicos contribuye a que la inmigración magrebí sea percibida como una amenaza por los gobiernos y parte de la opinión pública de los Estados receptores. Esta preocupación va sin duda ligada a los cambios sufridos por el sistema internacional a finales de los años ochenta: el progresivo afianzamiento del fin de la guerra fría ha desplazado las tensiones producidas por el enfrentamiento Este-Oeste hacia el eje de tensión Norte-Sur, cargado de una gran variedad de "retos", "amenazas" y conflictos potenciales.

En este contexto geopolítico, la política de inmigración del gobierno español ha sufrido cambios fundamentales determinados por: a) el aumento del número de inmigrantes y las necesidades que impone el hecho de ser un nuevo país de inmigración; b) los compromisos comunitarios en materia de libre circulación de personas y fronteras externas; c) la insatisfacción provocada por la antigua política de inmigración, y d) el surgimiento de actirudes sociales de rechazo a los inmigrantes. Puesto que la limitación del número de inmigrantes se convierte en objetivo prioritario, se adoptan importantes medidas de política interna: refuerzo de los controles fronterizos, integración legal y social de algunos de los individuos ya inmigrados, y modificaciones normativas y administrativas. Pero todo ello es insuficiente por sí solo y necesita ser complementado con medidas de política exterior orientadas a atacar directamente las causas de la inmigración: favorecer el desarrollo económico y la estabilidad social $y$ política de los países emisores, tanto de forma bilateral como multilateral, en el marco de la Europa comunitaria y de foros diplomáticos mediterráneos.

\section{El estrechamiento de las relaciones económicas hispano-magrebíes}

Los máximos responsables de la política exterior española han sido muy conscientes del estrecho vinculo existente entre el desarrollo económico de los países emisores y la reducción de los movimientos migratorios. Así lo expresaba el fallecido ministro de Asuntos Exteriores, Fernández Ordónez (1991): «Sólo el desarrollo económico logrará evitar una masiva Ilegada a las playas europeas de estos nuevos "espaldas mojadas" "; y en el mismo sentido se pronunciaba el Secretario General de Política Exterior, Francisco Villar (1992: 686): «Frente a este importante reto, la principal medida a adoptar, a nuestro juicio [...], es el desarrollo de los países de origen de estos emigrantes, de forma que no se vean obligados a buscar otros mercados laborales [...]". Por ello no es de extrañar que desde el inicio de los años noventa fueran objetivos principales del Palacio de Santa Cruz "promover la prosperidad y la estabilidad" de los Estados magrebíes, así como "procurar el desarrollo económico y social de estas naciones", pues "es indudable que España busca "anclar" esas poblaciones del sur en sus países de origen", según el director general de Política Exterior para África y Medio Oriente, Miguel Ángel Moratinos (1991: 6-9).

Ahora bien, ¿en qué medida se han perseguido estos objetivos? Los intercambios comerciales hispano-magrebies aumentaron en términos absolutos entre 1985 y 1993 — de forma moderada, eso sí-, pero su peso relativo se 
vio considerablemente reducido. A este respecto, Argelia y Marruecos constituyeron una excepción, pues se han afianzado como mercados atractivos y con proyección de futuro para los productos españoles. Aunque la balanza comercial española con el Magreb ha sido negativa desde 1985, la tasa de cobertura española ha mejorado notablemente y hay una tendencia hacia el equilibrio entre exportaciones e importaciones. De todo ello no es posible inferir que en el terreno comercial haya habido un estrechamiento significativo de las relaciones hispano-magrebíes.

Por lo que respecta a las inversiones españolas en el Magreb, el ministro Javier Solana (1992: 638) manifestaba la necesidad de potenciarlas: "[...] si no somos capaces de permitir, cle subrayar y de alentar los flujos de capitales del Norte al Sur, nos encontraremos con migraciones, cada vez más intensas del Sur al Norte». En este sentido, las inversiones españolas directas autorizadas en el Magreb, y más concretamente en Marruecos, han aumentado espectacularmente desde 1989: mientias que en 1988 su valor era de unos 484 millones de pesetas, en 1993 la cifra ascendió hasta 17.038 millones de pesetas, con lo que España se convertía en el segundo inversor más importante en Marruecos, por detrás de Francia.

Por último, cabe señalar que la evolución española de la cooperación al desarrollo con destino al Magreb ha reflejado la voluntad expresada por los responsables de la política exterior española, pues aumentó progresivamente y de forma significativa: el monto de la AOD dirigida al Magreb, hacia Marruecos y Argelia fundamentalmente; los créditos FAD ototgados a Marruecos y Argelia; los programas y proyectos con Marruecos, Mauritania y Argelia; $y$, de forma general, el número de acuerdos de cooperación en ámbitos diversos (técnico-financiera, cientifica, tecnológica y cultural). Pero el volumen global de la cooperación española al desarrollo sigue siendo muy modesto.

\section{La política exterior y de seguridad española hacia el Magreb}

Además de la mencionada cooperación económica, para contribuir a la estabilidad de la orilla sur del Medoc y al uanclaje» de sus poblaciones, el instrumento utilizado por la política exterior española en el Magreb ha sido la institucionalización del diálogo político.

Eri el caso del principal foco de interés español, Marruecos, el comienzo formal de dicha institucionalización no tiene lugar hasta septiembre de 1989, con ocasión de la visita a España de Hassan II. Pero antes de esa fecha los temas de inmigración ya habían sido planteados en 1984, durante la preparación del Convenio de Asistencia Mutua entre España y Marruecos con vistas a prevenir, investigar y reprimir las infracciones aduaneras, texto que no entraría en vigor hasta 1991. Por otro lado, en 1988 ya había tenido lugar en Rabat la primera reunión de la Comisión Mixta jurídico-consular para abordar cuestiones relativas a la inmigración marroquí en España.

En la segunda reunión de la Comisión Mixta, celebrada en diciembre de 1990, España planteó a Marruecos la obligación de exigir visados a los magre- 
bíes como consecuencia de las exigencias del proceso Schengen en el ámbito comunitario. Contrariamente a lo esperado, Marruecos comprendió la obligación española, posiblemente a causa de la prioridad de los graves desórdenes internos que acaparaban la atención gubernamental y del agradecimiento que en esos diffciles momentos era debido a la visita de Felipe González. El requisito de visado para los nacionales de países del Magreb con destino a España se haría efectivo sin mayotes obstáculos: ninguno por parte de Libia, Mauritania y Túnez; Argelia aplicó la reciprocidad, y Marruecos sólo planteó algunos problemas técnicos, no políticos ${ }^{5}$. Por tanto, la exigencia de visado no perjudicó a las telaciones hispano-magrebies, y además en julio tuvo lugar la firma del Convenio de Arristad, Buena Vecindad y Cooperación con Marruecos, que significaba la adopción de un nuevo marco político privilegiado en un momento de fuerte inmigración marroquí en España.

Desde el punto de vista de las migraciones, 1992 fue un año crítico en las relaciones hispano-marroquíes. En febrero se firmó el Acuerdo HispanoMarroquí relativo a la circulación de personas, el tránsito y la readmisión de extranjeros entrados ilegalmente ${ }^{6}$. Y durante el verano se produjo la llamada "crisis de las pateras" (al menos 10.000 marroquíes cruzaron el estrecho de Gibraltar ilegalmente), que comportó cierto desprestigio internacional de Marruecos y el aumento de las presiones españolas para una actuación represiva eficaz; el monarca alauí se decidió finalmente a aplicar más controles fronterizos, un endurecimiento de las penas para los infractores y mayor coordinación policial, medidas éstas que hasta el momento actual han dado excelentes resultados y que fueron agradecidas por el entonces ministro de Interior español, José Luis Corcuera.

Las migraciones ilegales marroquies han sido, pues, parcialmente resueltas con una actuación diplomática bilateral, en el marco de lo que el Ministerio de Asuntos Exteriores ha definido como una concepción ampliada de la seguridad, es decir, un enfoque no exclusivamente defensivo dirigido a la creación de una red de intereses comunes y al fortalecimiento de la cooperación económica y política (Solana, 1992: 635).

\section{Ensayos diplomáticos multilaterales en el Mediterráneo}

El tratamiento bilateral de los movimientos migratorios mediterráneos es obviamente insuficiente debido a las causas y al carácter multilateral del fenómeno.

5. Por ejemplo, con respecto al precio del visado, a los casos de marroquíes empleados en barcos pesqueros espafioles, a los casos de matroquíes en tránsiro hacia otros palses comunitarios, etc.

6. La entrada en vigor del Acuerdo sería demorada por Marruecos en protesta al veto que el Parlamento Europeo impuso a la aprobación del protocolo financiero que preveĺa la entrega de 60.000 millones de peseras a Marruecos para los cinco años siguientes. Sucesivas visitas de los ministros de Asuntos Exteriores, Fernández Ordófrez y Javier Solana, en marzo y julio respectivamente, aplacaron el enojo marroquf y fueron aprovechadas para presionas sobre la ratificación marroquí del Acuerdo. 
Por ello, la cooperación por vías diplomáticas de Estado a Estado necesita ser complementada con otros marcos de actuación originales en su planteamiento, multilaterales en su participación y globales en su enfoque geográfico y temático.

Desde esta perspectiva se fraguó entre diciembre de 1989 y enero de 1990 la idea hispano-italiana de creat la Conferencia sobre Seguridad y Cooperación en el Mediterráneo (CSCM) con una metodología análoga a la de la CSCE, pero aplicada a la cuenca mediterránea, y el proyecto sería presentado oficialmente en septiembre de 1990. A través de los ámbitos materiales o "cestas" de la CSCM (político-militar, económica y humanitaria), se planteaba un tradeoff consistente en cooperación para el desarrollo del sur del Mediterráneo a cambio de estabilidad social y política. El ministro de Asuntos Exteriores, Fernández Ordónez (1992: 552), manifestaba al respecto que «aquí la previsibilidad del comportamiento es la que se compensa con los estímulos económicos", y se ha especulado incluso que, aunque no expuesto abiertamente, el trade-off consistía en estimular el desarrollo y el empleo en el Sur a cambio de menos emigración hacia el Norte, con lo que la inmigración magrebí habría sido una de las principales causas del lanzamiento del proyecto de la CSCM (Aliboni, 1991: 33).

Paralelamente a la promoción del proyecto hispano-italiano, Francia lanzó otro proyecto de diálogo político regional, aunque en el ámbito geográfico más reducido del Medoc, la Conferencia de Cooperación Regional en el Mediterráneo Occidental o Grupo $5+5$. Este foro supuso inicialmente una competencia para la CSCM al demostrar su mayor operatividad a corto y medio plazo, pero España lo apoyó sin reservas, pues muchos de los objetivos perseguidos eran coincidentes. Durante el corto camino recorrido por el Grupo $5+5$ llegó a plantearse en la reunión de Argel de 1992 un trade-off relativo a los movimientos migratorios, pero esta vez consistente en la mejora del trato social y económico para los inmigrantes instalados en Europa a cambio de colaboración magrebí para reducir la emigración hacia el norte del Mediterráneo.

Por último cabe mencionar, como foro de cooperación Norte-Sur que posiblemente tratará los movimientos migratorios, la Conferencia del Mediterráneo, cuya celebración está previsté durante la presidencia española de la Unión Europea en el segundo semestre de 1995. Retomando los objetivos y la metodología de la paralizada CSCM, la Conferencia del Mediterráneo contará con la participación de todos los Estados mediterráneos y de todos los miembros de la Unión Europea.

\section{El filtro de la Europa comunitaria}

Los lentos pero seguros avances en el camino de la integración europea representan un filtro por el que los Estados miembros han de pasar a la hora de abordar determinados temas que son, total o parcialmente, competencia comunitaria. Este es el caso de la inmigración externa, la procedente de países terceros no comunitarios, tratada profusamente por la Europa comunitaria. 
En Ia línea de los objetivos e instrumentos de su política exterior, España ha apoyado, cuando no lanzado, las iniciativas comunitarias para contribuir al desarrollo económico del Magreb. Tal fue el caso de la Política Mediterránea Renovada (PMR), que para los Países Terceros Mediterráneos (PTM), y en particular para el Magreb, comportaba fundamentalmente: a) el fomento de mayores inversiones europeas; b) el aumento de la cooperación financiera a través de los VI Protocolos financieros para el periodo 1992-1996 (de los cuales 1.072 millones de ecus estaban destinados a Argelia, Marruecos y Túnez), y c) la apertura comercial de los Estados comunitarios a las importaciones de manufacturas y productos agrícolas procedentes del sur del Mediterráneo. Con todo, la PMR ha decepcionado a las autoridades españolas porque desde 1989 los estados comunitarios no mediterráneos han centrado su atención en los países de la Europa central y oriental.

Desde la cumbre de Corfú de 1994 este desequilibrio en favor del Este parece empezar a ser compensado con medidas dirigidas al desarrollo del sur del Mediterráneo, como el anuncio de la celebración de la Conferencia del Mediterráneo en 1995 y la aprobación en octubre de 1994 de un paquete financiero de 5.500 millones de ecus para el periodo 1995-2000. El esfuerzo y la tenacidad de la diplomacia española, encabezada por Manuel Marín, comienzan pues a dar sus frutos, para lo cual parece haber sido uno de los principales argumentos el flujo migratorio procedente del sur del Mediterráneo.

Esta inmigración complica considerablemente la total consecución de la libertad de circulación de personas. En el ámbito estrictamente comunitario ni el Acta Única Europea (1986) ni el Tratado de la Unión Europea (1992) han proporcionado las herramientas normativas adecuadas para homogeneizar el tratamiento comunitario de la inmigración externa. Ha sido necesario por ello acudir a una actuación "paracomunitaria", de tipo intergubernamental, que permitiera esquivar los rigores formales de la Comunidad y la oposición firme de algunos Estados. Dos han sido las vías de actuación paracomunitarias:

a) Cooperación Política Europea, en el marco de la cual se ha desarrollado una intensa actividad organizada en numerosos grupos de trabajo y órganos de decisión, como el Grupo Trevi (para la cooperación en asuntos de interior), los ministros de la Inmigración y el Grupo ad boc Inmigración, el Grupo de Asistencia Mutua (GAM, para cooperación aduanera), y el Grupo especial de coordinadores o Grupo Especial (para coordinar en cada Estado miembro el conjunto de actividades relacionadas con la consecución de la libre circulación de personas), por citar los más importantes en el ámbito que nos ocupa. De la actuación de esta "grupologia" ha sido siempre partícipe la diplomacia española, y no en vano, el Grupo Trevi 92 fue creado durante la presidencia española en 1989 , periodo en el que también fue adoptado en Madrid el Documento de Palma, texto de gran relevancia que consensuaba un marco jurídico para el establecimiento de una frontera 
exterior común y la desaparición de las fronteras internas de la Europa comunitaria (Pastor Ridruejo, 1990: 8-9).

b) Proceso Schengen, al cual se han adherido aquellos Estados que desean adoptar una política común de inmigración suprimiendo los controles en las fronteras comunes para trasladarlos a las fronteras exteriores ${ }^{7}$. Este "proceso" surgió en 1985 de un acuerdo inicial sobre controles franco-alemanes, al cual se sumarían los miembros del Benelux en el Acuerdo de Schengen, un conjunto de declaraciones de intención cuyas medidas de aplicación serían definidas en el Convenio de aplicación del Acuerdo de Schengen, en 1990 (Blanc, 1991: 722-726). Schengen constituyb una fuite en avant provocada por el estancamiento de los trabajos en el ámbito comunitario, y uno de sus resultados ha sido la creación de facto de una doble velocidad en materia de política común de inmigración. Desde junio de 1991 España forma parte de la "primera velocidad" al firmar los Acuerdos de Schengen cuyo Convenio de aplicación ratificó en marzo de 1994. La entrada en vigor de éste tuvo lugar el 26 de marzo de 1995 -durante los tres meses siguientes se permite el establecimiento de controles transitorios- entre siete de los nueve Estados signatarios: Alemania, Bélgica, España, Francia, Luxemburgo, Países Bajos y Portugal. Los siguientes Estados en los que previsiblemente el Convenio entrará en vigor serán Austria, Italia y Grecia —estos dos últimos ya firmaron los acuerdos de Schengen en 1990 y 1992, respectivamente.

La integración española en las actuaciones comunitarias mencionadas ha comportado la adopción de medidas restrictivas para los ciudadanos magrebíes que posiblemente España no hubiera adoptado de forma unilateral, como es el caso de la exigencia de visados. Por ello podrfa parecer que las instancias comunitarias han constreñido la capacidad de actuación española en temas de inmigración, pero lo cierto es que estas obligaciones europeas han sido una oportuna coartada frente a la diplomacia magrebí. Al mismo tiempo, debe destacarse que la diplomacia española se ha convertido en el mejor valedor del Magreb en Europa, pues no ha escatimado esfuerzos para: a) sensibilizar a los otros Estados miembros de los problemas que afectan al Magreb; b) proponer iniciativas y apoyar esfuerzos de tipo económico y político en favor del sur del Medoc, y c) mediar en los conflictos surgidos entre instancias comunitarias y países del Magreb --por ejemplo, entre el Parlamento Europeo y Marruecos.

\section{Conclusiones}

El fin de la guerra fría ha puesto al descubierto desequilibrios que durante muchos años permanecieron latentes en el Mediterráneo. Los contrastes demo-

7. No han firmado los Acuerdos de Schengen: Austria, Dinamarca, Finłandia, Irlanda, Reino Unido, Suecia. 
gráficos, económicos y sociopolíticos que se dan entre las orillas norte y sur del Medoc han pasado a ser percibidos como una fuente de amenazas para los estados de la ribera norte: los movimientos migratorios Sur-Norte que tienen lugar en el Medoc son fruto de esos contrastes.

En España, la inmigración procedente del Magreb, compuesta predominantemente por marroquíes, es cuantitativamente poco importante, pero aun así la limitación del número de magrebíes que emigran hacia territorio español ha pasado a ser considerada un objetivo prioritario. Los instrumentos de política interna que pueden conseguir una reducción de la inmigración magrebí son, sin embargo, insuficientes e inadecuados por las características del fenómeno migratorio, y por ello cabe acudir a la política exterior como ámbito de actuación para hacer frente a la inmigración no deseada.

Los responsables de la política exterior española son plenamente conscientes de la necesidad de contribuir al desarrollo económico de los países emisores para "anclar" a esas poblaciones. Por ello resulta lógico que se hayan hecho esfuerzos considerables a través de la cooperación al desarrollo y del fomento de las inversiones españolas, principalmente en Marruecos, aunque los resultados son por ahora difícilmente evaluables. En el terreno diplomático España ha conseguido institucionalizar el diálogo político con los países del sur del Medoc, y de esta forma ha sido posible canalizar el tratamiento de las cuestiones migratorias y de los problemas a ellas referidos.

Por otro lado, esta actuación bilateral ha necesitado ser complementada a nivel multilateral para implicar a otros actores estatales con problemas comunes en materia de inmigración. De ahí el lanzamiento del proyecto hispanoitaliano de la CSCM, en parte motivado por la necesidad de plantear un trade-off consistente en desarrolio para el sur del Mediterráneo a cambio de menos emigración hacia el norte. La prosperidad económica y la estabilidad sociopolítica de los países del Magreb también llevaron a España a apoyar los trabajos del Grupo 5+5, y con una lógica similar se dispone a organizar la Conferencia del Mediterráneo en 1995. No es de extrañar, por tanto, que en el ámbito de la Europa comunitaria España se haya convertido en la principal valedora de los intereses magrebíes. Eso sí, sin descolgarse de la uprimera velocidad" que en materia de inmigración y fronteras exteriores constituye el espacio Schengen.

A la vista de los hechos es difícil dudar de la influencia que la inmigración magrebí ha tenido sobre la política exterior española, sin que ello deba interpretarse como un condicionamiento total de las relaciones hispano-magrebies a las cuestiones migratorias. Dentro de la concepción ampliada de la seguridad que guía la actuación de los responsables diplomáticos españoles, los movimientos migratorios no pueden ser desligados del contexto social, económico y político en el que surgen. Desde esta perspectiva, el interés español consiste en actuar de forma realista y con visión de futuro, es decir, contribuyendo a la eliminación de las causas de la inmigración magtebí. Contrariamente a lo que suele suceder, solidaridad y egoísmo se conjugan desde el momento en que los intereses españoles y magrebíes devienen comunes. 


\section{Bibliografía citada}

Aliboni, Roberto (1991). "European Security across the Mediterranean". Chaillot Papers, núm. 2. París: Institute for Security Sudies, UEO.

BLANC, Hubert (1991). "Schengen: le chemin de la libre circulation en Europe". Rerue du Marché Commun et de l'Union Européenne, núm. 351, p. 722-726.

COLECTIVO IOE (1987). "Los inmigrantes en España». Documentación social núm. 66.

- (1992), "La inmigración magrebi en España", en LOPEZ GARCiA, Bernabé y orros. España-Magreb, siglo XXI. Madrid: Mapfre, p. 233-247.

DUCHACEK, Ivo D. (1988). "Multicommunal and Bicommunal Polities and Their International Relations", en DUCHACEK, Ivo D.; LATOUCHE, Daniel; STEVENSON, Garth. Perforated Sovereignties and International Relations, Westport-Connecticut: Greenword Press.

Eurostat (1991). Demographic Statistics 1991. Bruselas: Comision de las Comunidades Europeas.

- (1993). Demographic Statistics 1993. Bruselas: Comisión de las Comunidades Europeas.

Fernandez OrdoñzZ, Francisco (1990). "Comparecencia del Ministro de Asuntos Exteriores, don Francisco Fernández Ordóñez, ante la Comisión de Asuntos Exteriores del Senado, para informar sobre la situación del Mediterráneo y en particular en el Golfo y sobre la reunión de la CSCE en París", en OID. Actividades, textos y documentos de la Política Exterior española 1990. Madrid: Ministerio de AA.EE., p. $550-560$.

- (1991). Hacia una nueva concepción del Mediterráneo (conferencia pronunciada en la Universidad Internacional Menéndez y Pelayo el 15 de julio de 1991). Madrid: Ministerio de AA.EE.

Garcia Segura, Caterina (1993). "La evolución del concepto de actor en la Teoría de las Relaciones Internacionales». Papers, núm. 41, p. 13-31.

IsOPLAN (1991). "L'immigration des ressortissants de pays tiers dans les États méridionaux de la Communauté Européenne", en Europe Sociale. Bruselas: Comisión de las Comunidades Europeas.

Moratinos CuYaube, Miguel Ángel (1991). Politica exterior y de cooperación en el Magreb (Informativo 8). Madrid: Dirección General de Política Exterior para África y Medio Otiente, Ministerio de AA.EE.

PASTOR RidRUEJO, Rafael (1990), "La desaparición de las fronteras en la Europa comunitaria y los problemas demográficos e inmigratoriosn. Información Comercial Española, núm. 683, p. 7-13.

SOLANa Madariaga, Javier (1992). "Comparecencia del Ministro de Asuntos Exteriores, Javier Solana Madariaga, ante la Comisión de Asuntos Exteriores del Senado, para informar sobre la política española con relación al Magrebn, en OID. Actividades, textos y documentos de la Politica Exterior española 1992. Madrid: Ministerio de AA.EE., p. 631-647.

VILLAR y ORTIZ DE URBINA, Francisco (1992). «Comparecencia del Secretario General de Política Exterior, Don Francisco Villar y Ortiz de Urbina, ante la Comisión de Asuntos Exteriores del Congreso de los Diputados, para informar del estado de las relaciones con los países del Magreb", en OID. Actividades, textos y documentos de la Politica Exterior española 1992. Madrid: Ministerio de AA.EE., p. 685-686.

WEINER, Myron (1992). "Security, Stabilizy and International Migration". International Security, vol. 17, núm. 3, p. 91-126. 